\section{Sending ammonia signals}

\section{By Steve Edelson, Executive Editor}

Ammonia has long been viewed as a toxic cellular by-product of glutamine metabolism that has little or no functionality. New findings by a group at Pfizer Inc. now suggest this may not always be the case. The team found that in tumors, ammonia functions as a diffusible signal that can trigger autophagy in neighboring cancer cells, which enables them to be better prepared to counter external stressors such as chemotherapeutics. ${ }^{1}$

The results suggest that disrupting glutamine metabolism to reduce ammonia could be explored as a therapeutic strategy. The challenge will be figuring out how to therapeutically target the underlying pathways.

In the presence of oxygen, normal cells extract as much energy as possible from glucose primarily through mitochondrial oxidative phosphorylation. In contrast, the majority of tumors grow mostly through glycolysis, a less efficient but faster process that involves converting glucose to lactate in the cytosol.

As a result, the mitochondria of tumor cells are not used to generate ATP - instead, they work overtime producing intermediates for the synthesis of nucleic and fatty acids via the tricarboxylic acid (TCA) cycle (see Figure 1, "Ammonia as a stress signal in cancer").

One of the substrates for the TCA cycle, $\alpha$-ketoglutarate, is generated from glutamine in the mitochondria following two deamination reactions. As a by-product, two molecules of ammonia are released.

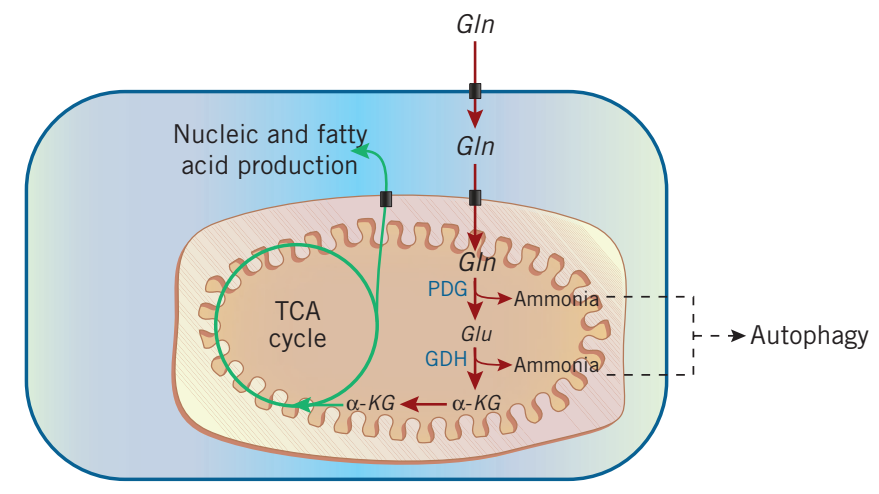

The Pfizer team found that this ammonia may fulfill a signaling function among cancer cells.

In cell lines, the researchers showed that ammonia triggered autophagy, a process by which cells degrade their own cytosolic components to prolong cell survival. Moreover, an analysis of human tumors showed that their interstitial fluids "contained ammonia at concentrations comparable to those found to trigger autophagy in vitro," the researchers wrote in their paper, published in Science Signaling.

The upshot, according to the paper, is that "ammonia is not simply a toxic metabolite as thought previously, but also a biologically active molecule that stimulates autophagy, a major stress-response pathway in tumor cells."

\section{Getting away from mTOR}

The only approved drug known to increase autophagy in preclinical studies is Rapamune sirolimus, a mammalian target of rapamycin (mTOR; FRAP; RAFT1) inhibitor that Pfizer's Wyeth unit markets as an immunosuppressant to prevent organ transplant rejection. Indeed, the link between mTOR and autophagy was the impetus for the recent research.

"We were interested in autophagy because of the connection between that process and mTOR - the work was done when we were at Wyeth, which had a strong program in mTOR inhibitors," said Christina Eng, lead author on the paper. Eng is now a senior scientist at Pfizer, which acquired Wyeth last year.

"As we worked with reagents and cell lines, we made the observation that prolonged cell culture induced a dramatic upregulation of autophagy. I decided to follow up on why this is," which led to the discovery of ammonia as a potential signaling molecule for autophagy, said Eng.

"In the end, it turned out to be mTOR independent. It deviated from our original goals, but it's an interesting observation," she said.

mTOR-independent ways of modulating autophagy are important, as they should avoid interfering with the immune system.

Figure 1. Ammonia as a stress signal in cancer. Ammonia has long been seen as a toxic by-product of glutamine (Gln) metabolism in mitochondria. However, researchers from Pfizer Inc. have now shown that in cancer, ammonia produced by the tumor cells can actually trigger autophagy in neighboring cancer cells, which helps the cells protect themselves from environmental stressors such as chemotherapeutics. Ammonia is produced in mitochondria as a byproduct of two consecutive deamination reactions of Gln. First, Gln is converted into glutamate (Glu) by phosphate-dependent glutaminase (PDG); then, Glu is converted into $\alpha$-ketoglutarate $(\alpha-K G)$ by glutamate dehydrogenase (GDH). $\alpha-K G$ then enters the tricarboxylic acid (TCA) cycle, leading to nucleic and fatty acid production. 


\section{ANALYSIS}

\section{TARGETS \& MECHANISMS}

In this case, the results suggest that the glutamine pathway could represent an mTOR-independent target and that antagonizing the pathway could be useful in cancer.

"If you could target glutamine metabolism, one outcome might be to block ammonia," said Eng. "We speculate that ammonia is a diffusible signal that tells neighboring cancer cells to protect themselves in an environment of stress. If you could block that, you may cause the neighboring cells to be more sensitive to different types of stress," including chemotherapy.

Alternatively, boosting glutamine levels could help upregulate autophagy in neurodegenerative disorders such as Huntington's disease and Parkinson's disease. In those diseases, ramping up autophagy represents a potential therapeutic strategy because it can lead to the destruction of disease-associated toxic protein aggregates. ${ }^{2}$

According to Eng, the next major question is understanding the precise mechanism by which ammonia triggers the start of autophagy. But that work will likely have to be done by others. Pfizer has not patented the findings described in the Science Signaling paper and does not have programs targeting ammonia.

Edelson, S. SciBX 3(19); doi:10.1038/scibx.2010.578

Published online May 13, 2010

REFERENCES

1. Eng, C.H. et al. Sci. Signal.; published online April 27, 2010; doi:10.1126/scisignal.2000911

Contact: Robert Abraham, Pfizer Inc., New York, N.Y. e-mail: Robert.Abraham@pfizer.com

2. Floto, R.A. et al. Autophagy 3, 620-622 (2007)

COMPANIES AND INSTITUTIONS MENTIONED

Pfizer Inc. (NYSE:PFE), New York, N.Y.

Wyeth (NYSE:WYE), Madison, N.J. 